\title{
NON-INTRUSIVE MEASUREMENT OF AXIAL ELECTRIC FIELDS IN LOW-PRESSURE GLOW DISCHARGES BY VELOCITY MODULATION LASER SPECTROSCOPY
}

\author{
Michael B. RADUNSKY and Richard J. SAYKALLY \\ Department of Chemistry. University of California. Berkeley, Berkeley, CA 9.4720, USA \\ Received 9 August 1988; in final form 2 September 1988
}

\begin{abstract}
High resolution (Doppler-sesolved) measurement of Doppler-shifted ion absorption frequencies allows the determination of the electric field responsible for the shift. A new method based on velocity modulation laser spectroscopy is described for the nonperturbative measurement of the axial electric fields in glow discharges. The $\mathrm{A}^{2} \Pi_{\mathrm{u}} \leftarrow \mathrm{X}^{2} \Sigma_{\mathrm{g}}^{+}(4,0)$ band of $\mathrm{N}_{2}^{+}$has been used as a probe of a He/N, discharge. Half-wave rectification of a sine-wave-driven discharge allows observation of Doppler shifts in the ion transition frequencies since the ions are traveling in only one dircction with respect to the laser wavevector. Electric fields and $E / N$ values have been measured at pressures from 3 to 11 Torr. Fields in the range of $10-15 \mathrm{~V} / \mathrm{cm}$ were observed.
\end{abstract}

\section{Introduction}

The average macroscopic electric field that exists in a glow discharge is undoubtedly one of its most important properties, influencing or determining many of its other characteristics. For example, the total translational energy content of the ions in the plasma is a strong function of $E / N$ [1]. The fact that plasma electric fields are notoriously difficult to measure in a manner that does not alter the plasma itself has been one of the principal obstacles in developing a quantitative understanding of electrical discharges.

Traditionally, Langmuir probes have been used to measure plasma fields, but these suffer from perturbations by sheath accumulation. Non-intrusive measurements of the electric fields through observation of Stark effects with laser spectroscopy have been demonstrated by several groups. Moore et al. [2] measured the electric field in the sheath region of a $50 \mathrm{kHz} \mathrm{BCl}{ }_{3}$ discharge by observing the Stark mixing in electronic transitions of $\mathrm{BCl}$. The large field that exists in the sheath $(5 \mathrm{kV} / \mathrm{cm})$ made this observation possible. A similar attempt by Pan [3] to measure the electric field in the positive column of a glow discharge via the Stark effect of $\mathrm{CH}_{3} \mathrm{~F}$ was unsuccessful. By using sub-Doppler laser polarization spectroscopy, Wieman and Hänsch [4] observed Stark splittings in the $n=4 \leftarrow 2$ transition of deuterium atoms in the positive column of an $\mathrm{H}_{2}$ discharge. The value of the electric field observed in their experiment was $10 \mathrm{~V} / \mathrm{cm}$, in good agreement with the results obtained in the present work. Ganguly and Garscadden [5] observed Stark broadening of Rydberg transitions of He in order to measure the electric fields in glow discharges, and were able to determine the radial variation in the field with a precision of about $1 \mathrm{~V} / \mathrm{cm}$. Finally, Haese et al. [6] performed an experiment exploiting the Doppler shift of $\mathrm{ArH}^{+}$vibration-rotation transitions as a result of the axial electric field in a dc discharge. Instead of calculating the field, they chose to actually measure it with a Langmuir probe, and with this field value to compute the mobility of the ion. It is important to note that the methods based on the observation of the Stark effect or polarization effects sample the total electric field experienced by the molecule, including both the axial (drift) and the radial (ambipolar) field as well as interference from collisional fields. Doppler shift measurements, however, are sensitive only to the axial field, and hence offer a means for distinguishing between the two components of the average electric field.

In this paper we present a method for separately 
determining the axial electric field in low field regions $(\leqslant 15 \mathrm{~V} / \mathrm{cm})$ of discharges by the measurement of the ficld-induced Doppler shifts in the absorption frequencies of molecular ion transitions. By half-wave rectification of a low frequency $(25$ $\mathrm{kHz}$ ) discharge, current flows in only one direction with respect to the laser wavevector and only one velocity sideband of the shifted absorption frequency is observed. The intrinsic Doppler-broadened lineshape for the transition can thus be recorded, as well as the absolute Doppler shift of the transition. The disadvantage of this technique is that neutral transitions are also modulated, and therefore can interfere with the ionic spectrum (in this case, the first positive band system of $\mathrm{N}_{2}$ ). The main advantages, in addition to the axial field selectivity, are that the spectrum requires only Doppler-limited resolution, and no Stark splitting or broadening need be observed, greatly reducing both the experimental and theoretical complexity of the measurement. In addition, there is no interference from fields generated by the local ion distribution [7].

The technique described here is a modification of the method of velocity modulation developed in this laboratory [8]. In part, this work was undertaken to more fully understand the lineshapes obtained in the velocity modulation experiment. The observation of only the velocity modulation lineshape leaves the Doppler shift and width of the transition inextricably mixed, i.e. the depth of modulation is unknown [9]. Therefore it is difficult to extract accurate translational temperatures and/or pressure broadening parameters from this method. The present experiment permits the observation of the true lineshape, and as a result of this fact, facilitates the determination of these important parameters.

\section{Experimental}

The dye laser spectrometer used in these experiments is essentially the same as the apparatus described in conjunction with the velocity modulation study of the $A^{2} \Pi_{u}-X^{2} \Sigma_{g}^{+}(7,3)$ hand of $N_{2}^{+}$reported earlier [10]. Briefly, the instrument consists of a tunable dye laser (Coherent CR 699-29) dual beam absorption spectrometer. A $1 \mathrm{~m}$ long watercooled velocity modulation discharge cell with sidearm electrodes is used as a sample cell. The sidearms ensure that only the positive column of the discharge is probed.

The principal modification is the introduction of a high power diode (Unitrode HVH 10000) in series with the discharge. This results in half-wave rectification of the discharge current waveform. The rectified waveform is not significantly distorted from that of the unrectified discharge. The discharge is stable and no striations are visible (striations are known to cause field inhomogeneities). The second modification is the use of an ENI Plasmaloc II power supply to drive the discharge. This allows for higher operation frequency $(25 \mathrm{kHz})$ and smoother power delivery, with a corrcsponding increase in the discharge stability. This results in higher signal-to-noise ratios and much flatter baselines.

The $(4,0)$ band of the $A^{2} \Pi_{u} \leftarrow X^{2} \Sigma_{B}^{+}$system of $\mathrm{N}_{2}^{+}$was monitored in this experiment. This band was originally observed in high resolution by Grieman et al. [11] and the subsequent rotational assignments of Miller et al. [12] were used as a guide in this experiment. The $(4,0)$ bandhead is at $16329.490 \mathrm{~cm}^{-1}$ and therefore rhodamine $6 \mathrm{G}$ was the dye employed.

In all measurements the partial pressure of $\mathrm{N}_{2}$ was about $30 \mathrm{mTorr}$ and the balance was He. The dc current through the discharge was maintained at 120.0 $\mathrm{mA}$ by monitoring the voltage across a $0.5 \Omega$ resistor in series with the discharge. Each transition frequency was referenced to an etalon fringe (FSR $=\mathbf{6}$ $\mathrm{GHz}$ ). Both the red-and blue-shifted components of each transition were observed, and the Doppler shift was taken to be one half of the difference between them (fig. 1).

The first positive band system of neutral $N_{2}$ has strong transitions in the same spectral region as the $(4,0)$ band of $N_{2}^{+}$[13]. This crowding of the spectrum was the major source of error in the determination of the absorption shifts and we estimate this error to be about $0.002 \mathrm{~cm}^{-1}$.

\section{Theory}

In a standard velocity modulation experiment [8] the signal appears as an approximate first derivative lineshape. Without additional information it is impossible to separate the Doppler shift due to the elec- 


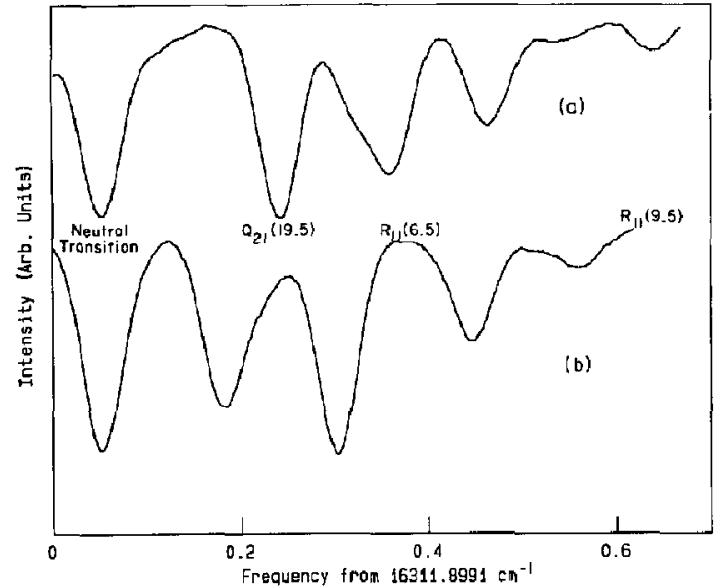

Fig. 1. Transitions observed with the discharge diode facing such that the ion current is (a) parallel to and (b) anti-parallel to the laser propagation direction showing the Doppler shift in transition frequency due to the axial electric field.

tric field acting on the ion and the inhomogeneous linewidth of the Doppler-broadened transition. Halfwave rectification of the discharge limits the directed motion of ions to only one direction with respect to the laser wavevector. The result is that upon lock-in demodulation of the laser absorption signal an unbroadened Gaussian lineshape is observed, shifted either to the red or the blue depending on the direction of current flow with respect to the laser beam.

The calculation of the electric field from the Doppler shift is straightforward as long as the ion mobility is independent of the electric field (low field limit, $\leqslant 25 \mathrm{Td}$ ) [1] as considered below. The Doppler shift in absorption frequency $(\delta p)$ caused by the ion drift velocity $\left(v_{\mathrm{D}}\right)$ is $\delta \nu=\frac{v_{\mathrm{D}}}{c} \nu$.

In the low field limit the ion drift velocity is proportional to the electric field [1]

$v_{\mathrm{D}}=K_{0} E$.

The proportionality constant is the mobility; for $\mathrm{N}_{2}^{+}$this is obtained experimentally from the work of Johnsen et al. [14] and Moseley et al. [15] and must be adjusted for temperature and pressure from the reduced mobility $\left(K_{0}\right)$ :

$K=K_{0} \frac{T}{273.15 \mathrm{~K}} \frac{760 \mathrm{~T}}{P} \mathrm{~cm}^{2} / \mathrm{V} \mathrm{s}$.

In addition $K_{0}$ must be corrected for gas composition,

$\frac{1}{K_{0, \operatorname{mix}}}=\sum \frac{X_{i}}{K_{0, i}}$,

where $X_{i}$ is the mole fraction of component $i$. Combining these equations yields

$E=\frac{\delta \nu}{\nu} \frac{c}{K_{0, \operatorname{mix}}} \frac{P}{760 \mathrm{~T}} \frac{273.15 \mathrm{~K}}{T}$.

The temperature appearing in eqs. (3) and (5) is the gas kinetic temperature and is obtained here from the widths of neutral $\mathrm{N}_{2}$ transitions in the same spectral region. From eq. (5) and the shifts measured for the $(4,0)$ band, the axial electric field in the positive column of the discharge is calculated.

\section{Results}

The observed Doppler shifts for the $(4,0)$ band and the calculated electric fields are listed in table 1.

Values of measured and calculated parameters for $(4,0)$ band of $\mathrm{N}_{2}^{+}$. (Numbers in parentheses are $1 \sigma$ errors)

\begin{tabular}{|c|c|c|c|c|}
\hline$P($ Torr $)$ & $\delta \nu\left(\mathrm{cm}^{-1}\right)$ & $v_{\mathrm{D}}(\mathrm{m} / \mathrm{s})$ & $E(\mathrm{~V} / \mathrm{cm})$ & $E / N(\mathrm{Td})$ \\
\hline 3 & $0.0349(27)$ & $698(54)$ & $10.9(1.0)$ & $15.0(1.2)$ \\
\hline 4 & $0.0312(24)$ & $624(48)$ & $11.7(1.5)$ & $13.1(1.0)$ \\
\hline 5 & $0.0271(20)$ & $542(40)$ & $13.8(1.4)$ & $11.2(0.8)$ \\
\hline 6 & $0.0268(13)$ & $536(26)$ & $14.3(1.2)$ & $10.8(0.5)$ \\
\hline 7 & $0.0226(11)$ & $452(22)$ & $13.8(1.4)$ & $9.1(0.4)$ \\
\hline 9 & $0.0178(15)$ & $357(29)$ & $14.0(1.6)$ & $7.1(0.6)$ \\
\hline 10 & $0.0152(13)$ & $304(26)$ & $12.5(1.7)$ & $6.1(0.5)$ \\
\hline 11 & $0.0128(19)$ & $256(38)$ & $10.9(2.0)$ & $5.1(0.8)$ \\
\hline
\end{tabular}




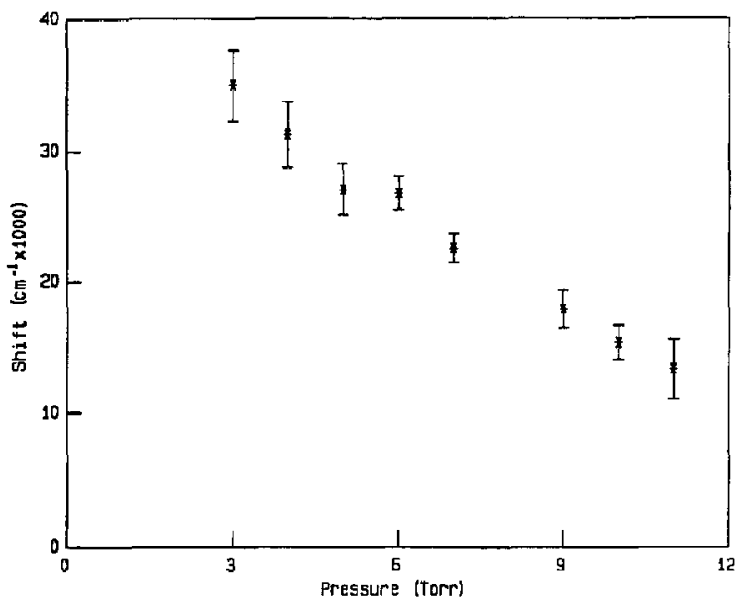

Fig. 2. Plot of Doppler shift in ion transition frequency as a function of pressure in discharge tube. (Error is $1 \sigma$.)

The shift is found to decrease approximately linearly with increasing pressure (fig. 2), reflecting the dependence of the ion mobility on collision frequency. The monotonic decrease in shift with pressure does not entirely agree with the trend observed by Haese et al. [6]. In a low pressure He discharge ( $<3$ Torr) they observed that the shift first increased with pressure, and then slowly fell off for pressures above 3 Torr. As suggested by the authors, this initial rise in the shifts is probably due to proton exchange of $\mathrm{ArH}^{+}$ with essentially stationary Ar atoms at higher Ar mole fraction (lower pressure). At higher total pressures collisions with Ar are less probable and the shift decreases with pressure as expected. This is in accord with our obscrvation and with cq. (3).

A plot of the plasma electric field versus pressure is shown in fig. 3. An average field value of $12.7 \pm 1.4$ $\mathrm{V} / \mathrm{cm}$ is obtained. The electric field is fairly constant with pressure, but there is a slight maximum in the field $(14.3 \pm 1.2 \mathrm{~V} / \mathrm{cm})$ at 6 Torr. Since the product of $n_{\mathrm{e}}$ and $E$ is expected to remain constant and since the current was held constant in our experiments no pressure variation in the field would be expected. A plot of $E / N$ versus pressure (fig. 4 ) indicates that the ion energy decreases with increasing pressure due to the larger number of energy sharing collisions. The maximum value of $E / N$ at 3 Torr of $15.0 \pm 1.2 \mathrm{Td}$ ( $1 \mathrm{Td}=10^{-17} \mathrm{~V} \mathrm{~cm}^{2}$ ) ensures that all measurements are in the low field limit and that eq. (2) is valid. Initially there appears to be a contradiction between the concept that $E / N$ determines the average ion en-

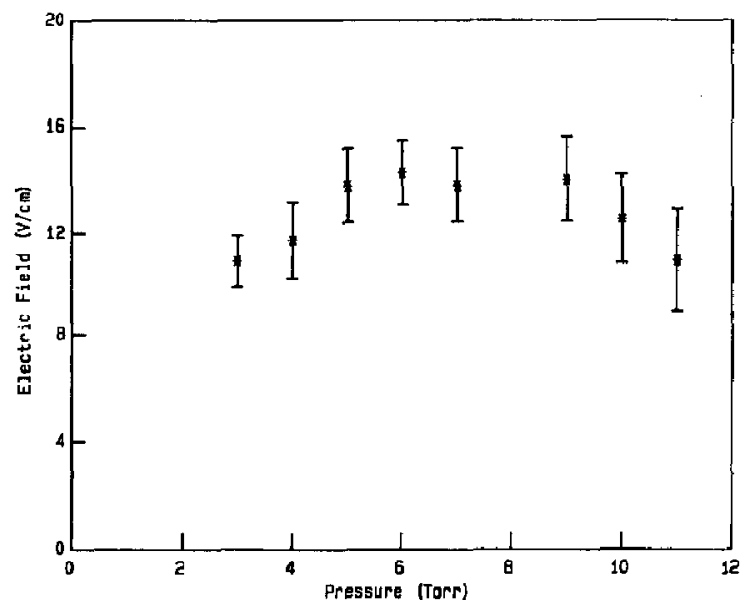

Fig. 3. Plot of axial electric field versus pressure.

ergy and the observed ion translational temperature, as derived from the measured linewidths in that the linewidths increase with pressure while $E / N$ decreases with pressure. However, the tolal ion energy includes the directed component of its velocity $\left(v_{\mathrm{D}}\right)$ as well as the random component. When this is taken into consideration the total ion energy decreases with pressure as does $E / N$.

The depth of modulation (i.e. the ratio of the Doppler shift, $\delta \nu$, to the Doppler width, $\Delta \nu$ ) decreases linearly with pressure (fig. 5). This reflects both the decrease in mobility and the increase in translational temperature with increasing pressure. This variation in modulation depth makes reliable

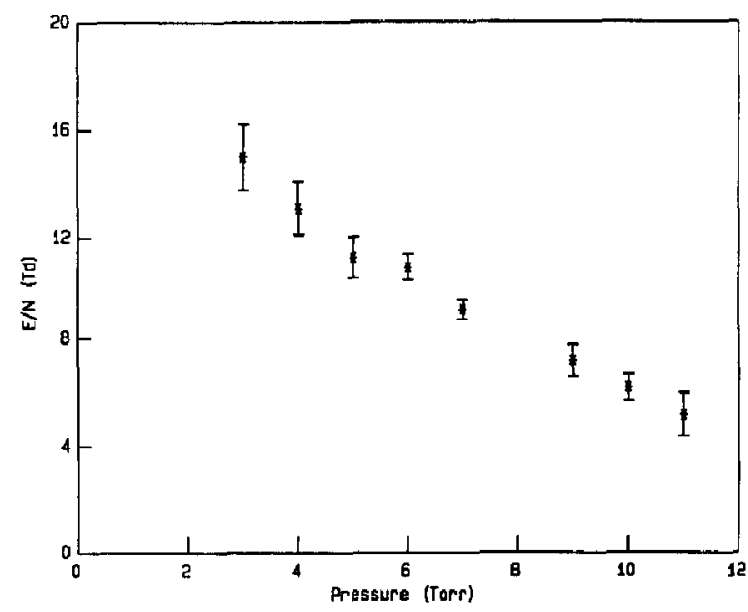

Fig. 4. Plot of electric field normalized to number density versus pressure. $\left(1 \mathrm{Td}=10^{-17} \mathrm{~V} \mathrm{~cm}^{2}\right)$. 


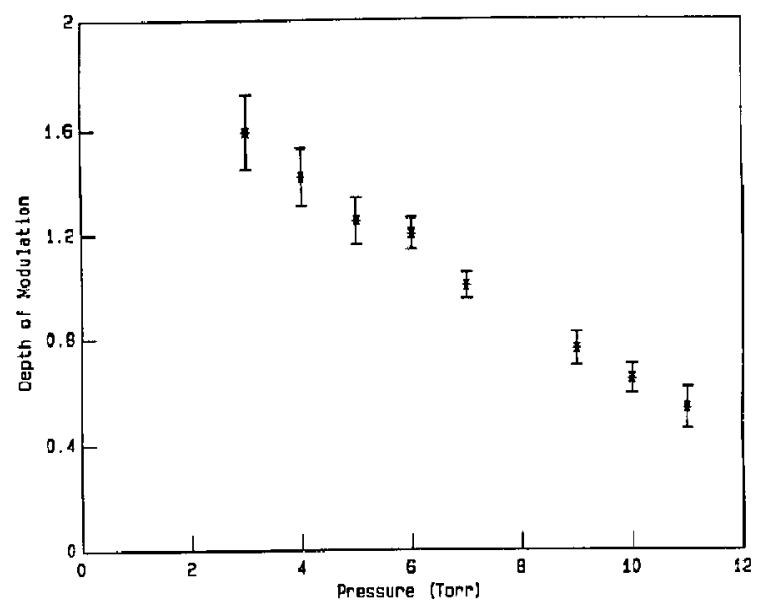

Fig. 5. Depth of modulation (ratio of Doppler shift to Doppler width ) plotted as a function of pressure.

measurement of linewidths difficult in a velocity modulation experiment. According to ref. [9] there exists a simple relationship between the pcak-to-peak separation of the apparent first derivative velocity modulation signal and the actual Doppler width of the transition only if this ratio, $\delta \nu / \Delta \nu$, is less than about 0.7. From fig. 5 it is apparent that only at pressures above 10 Torr can this simplification be made for the present system. Since most velocity modulation experiments have been performed in the 1-5 Torr region [8], an iterative solution to an implicit equation would be required in most cases. The use of such a procedure was shown to result in highly correlated shift and width determinations [9].

\section{Conclusions}

By measuring the Doppler shift in the frequency of molecular ion rovibronic transitions we have determined the axial electric field in the positive column of a $\mathrm{He} / \mathrm{N}_{2}$ discharge. The electric field was found to be a slowly varying function of pressure with a maximum at 6 Torr. The field values observed are consistent with those measured by other methods [57,9]. By separating the measurement of the Doppler shift due to the electric field and the width of the transition it is possible to measure translational temperatures and/or pressure broadening coefficients without resorting to an iterative procedure. At visible wavelengths the linewidth is entirely due to inhomogeneous broadening, and we have determined accurate translational temperatures for $\mathrm{N}_{2}^{+}$. The rise in the translational temperature of the ion with increasing pressure reflects the increased randomization of field energy with increased collision frequency.

\section{Acknowledgement}

We would like to acknowledge helpful discussions with Professor David Graves. This work was supported by the National Science Foundation (Grant No. CHE-8612296) and by the Imperial Chemical Industries Corporation. MBR was supported in part by a California Micro-Electronics Innovation and Computer Research Opportunities fellowship.

\section{References}

[1] E.W. McDaniel and E.A. Mason, The mobility and diffusion of ions in gases (Wiley-Interscience, New York, 1973).

[2] C.A. Moore, G.P. Davis and R.A. Gottscho. Phys. Rev. Letters 52 (1984) 538 .

[3] F.S. Pan, Ph.D. Dissertation, University of Chicago (1987).

14 C. Wieman and T.W. Hänsch, Phys. Rev. Letters 36 (1976) 1170 .

[5] B.N. Ganguly and A. Garscadden. Appl. Phys. Letters 46 (1945) 540 .

[6] N.N. Haese, F.S. Pan and T. Oka, Phys. Rev. Letlers 50 (1983) 1575 .

[7] R.C. Wouls, 42nd Sym posium on Molecular Spectroscopy, Columbus, Ohio (1987).

[8] C.S. Gudeman and R.J. Saykally, Ann. Rev. Phys. Chem. 35 (1984) 387.

[9] C.C. Martner, Ph,D. Dissertation, University of California, Bcrkcley (1986).

[10] M.B. Radunsky and R.J Saykally, J. Chem. Phys. 87 (1987) 898

[11] F.J. Grieman, J.C. Hansen and J.T. Moseley, Chem. Phys. Letters 85 (1982) 53 .

[12] T.A. Miller, T. Suzuki and E. Hirota, J. Chem. Phys. 80 (1984) 4671

[13] R.W.B. Pierce and A.G. Gaydon. The identification of molecular spectra (Wiley, New York, 1976).

[ 14$]$ R. Johnsen, H.L. Brown and M.A. Biondi, J. Chem. Phys. $52(1970) 5080$.

[15]J.T. Moscley, R.M. Snuggs, D.W. Martin and E.A. McDaniel. Phys. Rev. 78 ( 1969$) 234$. 